\title{
Nonlinear Nonhomogeneous Obstacle Problems with Multivalued Convection Term
}

\author{
Shengda Zeng ${ }^{1,2}$ (D) Yunru Bai ${ }^{3}$. Leszek Gasiński ${ }^{4}$
}

Received: 9 April 2021 / Accepted: 27 July 2021 / Published online: 12 January 2022

(C) The Author(s) 2021

\begin{abstract}
In this paper, a nonlinear elliptic obstacle problem is studied. The nonlinear nonhomogeneous partial differential operator generalizes the notions of $p$-Laplacian while on the right hand side we have a multivalued convection term (i.e., a multivalued reaction term may depend also on the gradient of the solution). The main result of the paper provides existence of the solutions as well as bondedness and closedness of the set of weak solutions of the problem, under quite general assumptions on the data. The main tool of the paper is the surjectivity theorem for multivalued functions given by the sum of a maximal monotone multivalued operator and a bounded multivalued pseudomonotone one.
\end{abstract}

Keywords Nonhomogeneous partial differential operator $\cdot$ Multivalued convection term · Generalized pseudomonotone operators · Surjectivity theorem · Existence

Mathematics Subject Classification 35J20 · 35J25 $\cdot 35$ J60

Shengda Zeng

zengshengda@163.com

Yunru Bai

yunrubai@163.com

Leszek Gasiński

leszek.gasinski@up.krakow.pl

1 Guangxi Colleges and Universities Key Laboratory of Complex System Optimization and Big Data Processing, Yulin Normal University, Yulin 537000, People's Republic of China

2 Faculty of Mathematics and Computer Science, Jagiellonian University in Krakow, Lojasiewicza 6, 30-348 Krakow, Poland

3 Guangxi University of Science and Technology, Liuzhou 545006, Guangxi, China

4 Department of Mathematics, Pedagogical University of Cracow, Podchorazych 2, 30-084 Krakow, Poland 


\section{Introduction}

Let $\Omega \subseteq \mathbb{R}^{N}$ be a bounded domain with a Lipschitz-boundary $\partial \Omega$. In this paper, we study the following nonlinear nonhomogeneous elliptic problem with a multivalued convection term and under obstacle condition

$$
\left\{\begin{array}{l}
-\operatorname{div} a(x, \nabla u) \in f(x, u, \nabla u) \text { in } \Omega \\
u(x) \leq \Phi(x) \text { in } \Omega, \\
u=0 \text { on } \partial \Omega,
\end{array}\right.
$$

where $a: \bar{\Omega} \times \mathbb{R}^{N} \rightarrow \mathbb{R}^{N}$ is continuous, monotone with respect to the second variable and satisfies particular other growth conditions to be described later, reaction term $f: \Omega \times \mathbb{R} \times \mathbb{R}^{N} \rightarrow 2^{\mathbb{R}}$ is multivalued and depends on the gradient of the solution (which makes the problem nonvariational) and obstacle $\Phi: \Omega \rightarrow[0,+\infty]$ is a given function.

As the setting is general enough and hypotheses are mild and natural, we incorporate in our framework many differential operators, such as the $p$-Laplacian, the $(p, q)$ Laplacian (i.e., the sum of a $p$-Laplacian and a $q$-Laplacian) and the generalized $p$-mean curvature differential operator. The precise conditions set on the data will be formulated in Sect. 3.

For the nonlinear elliptic problems with gradient dependence we refer to the following papers: Averna-Motreanu-Tornatore [1], Bai [2], Bai-Gasiński-Papageorgiou [3], Faraci-Motreanu-Puglisi [4], Gasiński-Papageorgiou [5,6], Gasiński-Winkert [7], Motreanu-Motreanu-Moussaoui [8], Guarnotta-Marano-Motreanu [9], PapageorgiouRădulescu-Repovš [10], Faraci-Puglisi [11], Figueiredo-Madeira [12], PapageorgiouRădulescu-Repovš [13], Tanaka [14], Guarnotta-Marano [15], Liu-Motreanu-Zeng [16], Marano-Winkert [17], Araujo-Faria [18], Bai-Papageorgiou-Zeng [19]. None of the above papers deals with multivalued or obstacle problems. To the best of our knowledge, this is the first paper combining all these phenomena in one problem. The main tool in the proof of the existence result for problem (1.1) will be the surjectivity result due to Le [20] for multivalued mappings generated by the sum of a maximal monotone multivalued operator and a bounded multivalued pseudomonotone mapping.

Since (1.1) is an obstacle problem, the appropriate set in which we are looking for its solutions is the following one

$$
\left\{u \in W_{0}^{1, p}(\Omega) \mid u(x) \leq \Phi(x) \text { for a. a. } x \in \Omega\right\}
$$

with a given obstacle function $\Phi: \Omega \rightarrow \overline{\mathbb{R}}_{+}=[0, \infty]$. When $\Phi \equiv+\infty$, problem (1.1) becomes the following nonlinear problem with multivalued convection term

$$
\left\{\begin{array}{l}
-\operatorname{div} a(x, \nabla u) \in f(x, u, \nabla u) \text { in } \Omega \\
u=0 \text { on } \partial \Omega
\end{array}\right.
$$


(see e.g., [5,10,21,22]). In addition, when $f$ is a single-valued function, problem (1.1) reduces to

$$
\left\{\begin{array}{l}
-\operatorname{div} a(x, \nabla u)=f(x, u, \nabla u) \text { in } \Omega \\
u=0 \text { on } \partial \Omega
\end{array}\right.
$$

(see e.g., $[1,8,10])$.

Moreover, for problems with double phase operators (which include the case for the $(p, q)$-Laplacian) and multivalued terms (and also dealing with obstacle problems), we refer to Zeng-Gasiński-Winkert-Bai [23-25]. Finally, we mention that MingioneRădulescu [26] provided an overview of recent results concerning elliptic variational problems with nonstandard growth conditions and related to different kinds of nonuniformly elliptic operators.

\section{Preliminaries}

Let $\Omega$ be a bounded domain in $\mathbb{R}^{N}$ and let $1 \leq r \leq \infty$. In what follows, we denote by $L^{r}(\Omega)$ and $L^{r}\left(\Omega ; \mathbb{R}^{N}\right)$ the usual Lebesgue spaces endowed with the norm $\|\cdot\|_{r}$. Moreover, $W_{0}^{1, r}(\Omega)$ stands for the Sobolev space endowed with the norm

$$
\|u\|:=\|\nabla u\|_{r} \text { for all } u \in W_{0}^{1, r}(\Omega)
$$

Let us now consider the eigenvalue problem for the $r$-Laplacian with homogeneous Dirichlet boundary condition and $1<r<\infty$ which is defined by

$$
\left\{\begin{array}{l}
-\Delta_{r} u=\lambda|u|^{r-2} u \text { in } \Omega \\
u=0 \text { on } \partial \Omega
\end{array}\right.
$$

A number $\lambda \in \mathbb{R}$ is an eigenvalue of $\left(-\Delta_{r}, W_{0}^{1, r}(\Omega)\right)$ if problem (2.1) has a nontrivial solution $u \in W_{0}^{1, r}(\Omega)$ which is called an eigenfunction corresponding to the eigenvalue $\lambda$. We denote by $\sigma_{r}$ the set of eigenvalues of $\left(-\Delta_{r}, W_{0}^{1, r}(\Omega)\right)$. From Lê [27] we know that the set $\sigma_{r}$ has a smallest element $\lambda_{1, r}$ which is positive, isolated, simple and it can be variationally characterized through

$$
\lambda_{1, r}=\inf \left\{\frac{\|\nabla u\|_{r}^{r}}{\|u\|_{r}^{r}}: u \in W_{0}^{1, r}(\Omega), u \neq 0\right\} .
$$

For $s>1$ we denote by $s^{\prime}=\frac{s}{s-1}$ its conjugate, the inner product in $\mathbb{R}^{N}$ is denoted by $\cdot$ and the Euclidean norm of $\mathbb{R}^{N}$ by $|\cdot|$. Moreover, $\mathbb{R}_{+}=[0,+\infty)$ and the Lebesgue measure of a set $A$ in $\mathbb{R}^{N}$ is denoted by $|A|_{N}$.

As for the data $a$ of the problem (1.1) we assume that

$H(a)_{0}: a: \bar{\Omega} \times \mathbb{R}^{N} \rightarrow \mathbb{R}^{N}$ is a function such that $a(x, \xi)=a_{0}(x,|\xi|) \xi$ with $a_{0} \in$ $C\left(\bar{\Omega} \times \mathbb{R}_{+}\right)$for all $\xi \in \mathbb{R}^{N}$ and with $a_{0}(x, t)>0$ for all $x \in \bar{\Omega}$, all $t>0$. 
For the regularity of $a_{0}$ as well as its behavior at zero, we will assume the following $H(a)_{1}: a_{0} \in C^{1}(\bar{\Omega} \times(0, \infty)), t \mapsto t a_{0}(x, t)$ is strictly increasing in $(0, \infty)$, $\lim _{t \rightarrow 0^{+}} t a_{0}(x, t)=0$ for all $x \in \bar{\Omega}$ and

$$
\lim _{t \rightarrow 0^{+}} \frac{t a_{0}^{\prime}(x, t)}{a_{0}(x, t)}=c>-1 \text { for all } x \in \bar{\Omega}
$$

For the growth assumptions on $a$, we will exploit a function $\vartheta \in C^{1}(0, \infty)$ satisfying

$$
0<a_{1} \leq \frac{t \vartheta^{\prime}(t)}{\vartheta(t)} \leq a_{2} \text { and } a_{3} t^{p-1} \leq \vartheta(t) \leq a_{4}\left(t^{q-1}+t^{p-1}\right)
$$

for all $t>0$, with some constants $a_{1}, a_{2}, a_{3}, a_{4}>0$ and for $1<q<p<\infty$. Now we can state the growth of $a$ as follows

$H(a)_{2}$ : there exists $a_{5}>0$ such that

$$
\left|\nabla_{\xi} a(x, \xi)\right| \leq a_{5} \frac{\vartheta(|\xi|)}{|\xi|} \text { for all } x \in \bar{\Omega}, \xi \in \mathbb{R}^{N} \backslash\{0\}
$$

The above defined function $\vartheta$ will be also exploited in the next assumption guaranteeing coercivity-like behavior of the function $a$ $H(a)_{3}: \nabla_{\xi} a(x, \xi) y \cdot y \geq \frac{\vartheta(|\xi|)}{|\xi|}|y|^{2}$ for all $x \in \bar{\Omega}, \xi \in \mathbb{R}^{N} \backslash\{0\}$ and $y \in \mathbb{R}^{N}$.

Under the above hypotheses, we can state the following lemma in which we will summarize some properties of the function $a: \bar{\Omega} \times \mathbb{R}^{N} \rightarrow \mathbb{R}^{N}$ (see e.g., Bai-GasińskiPapageorgiou [3, Lemma 2.2]).

Lemma 2.1 If hypotheses $H(a)_{0}-H(a)_{3}$ hold, then:

(i) $a \in C\left(\bar{\Omega} \times \mathbb{R}^{N} ; \mathbb{R}^{N}\right) \cap C^{1}\left(\bar{\Omega} \times\left(\mathbb{R}^{N} \backslash\{0\}\right) ; \mathbb{R}^{N}\right)$ and for all $x \in \bar{\Omega}$ the map $\xi \rightarrow a(x, \xi)$ is continuous, strictly monotone (and so maximal monotone);

(ii) there exists $a_{6}>0$, such that

$$
|a(x, \xi)| \leq a_{6}\left(1+|\xi|^{p-1}\right) \text { for all } x \in \bar{\Omega} \text { and } \xi \in \mathbb{R}^{N}
$$

(iii) $a(x, \xi) \cdot \xi \geq \frac{a_{3}}{p-1}|\xi|^{p}$ for all $x \in \bar{\Omega}$ and $\xi \in \mathbb{R}^{N}$.

The nonlinear operator $A: W_{0}^{1, p}(\Omega) \rightarrow W_{0}^{1, p}(\Omega)^{*}$ defined by

$$
\langle A(u), \varphi\rangle=\int_{\Omega}(a(x, \nabla u), \nabla \varphi)_{\mathbb{R}^{N}} d x \text { for all } u, \varphi \in W_{0}^{1, p}(\Omega),
$$

possesses the following useful properties (see e.g., Gasiński-Papageorgiou [28]). 
Proposition 2.2 If hypotheses $H(a)_{0}-H(a)_{3}$ hold, then the operator A defined by (2.4) is bounded, monotone, continuous, hence maximal monotone and of type $\left(\mathrm{S}_{+}\right)$.

In the following example we indicate some operators fitting in our framework.

Example 2.3 In what follows for simplicity, we drop the dependence of the operators $a$ on $x$. All the following maps satisfy hypotheses $H(a)_{0}-H(a)_{3}$ :

(i) If $a(\xi)=|\xi|^{p-2} \xi$ with $1<p<\infty$, then the corresponding operator is the classical $p$-Laplacian

$$
\Delta_{p} u=\operatorname{div}\left(|\nabla u|^{p-2} \nabla u\right) \text { for all } u \in W^{1, p}(\Omega) .
$$

(ii) If $a(\xi)=|\xi|^{p-2} \xi+\mu|\xi|^{q-2} \xi$ with $1<q<p<\infty$ and $\mu>0$ then the corresponding operator is the so called weighted $(p, q)$-Laplacian defined by $\Delta_{p} u+\mu \Delta_{q} u$ for all $u \in W^{1, p}(\Omega)$.

(iii) If $a(\xi)=\left(1+|\xi|^{2}\right)^{\frac{p-2}{2}} \xi$ with $1<p<\infty$, then the corresponding operator represents the generalized $p$-mean curvature differential operator defined by

$$
\operatorname{div}\left[\left(1+|\nabla u|^{2}\right)^{\frac{p-2}{2}} \nabla u\right] \text { for all } u \in W^{1, p}(\Omega) .
$$

(iv) If $a(y)=|y|^{p-2} y+\frac{|y|^{p-2} y}{1+|y|^{p}}$ with $2<p<+\infty$, then the corresponding operator takes the form

$$
\Delta_{p} u+\operatorname{div}\left(\frac{|\nabla u|^{p-2} \nabla u}{1+|\nabla u|^{p}}\right) \text { for all } u \in W_{0}^{1, p}(\Omega),
$$

which arises in various problems of plasticity.

Next, let us recall the notions of pseudomonotonicity and generalized pseudomonotonicity for multivalued operators (see e.g., Gasiński-Papageorgiou [29, Definition 1.4.8]) which will be useful in the sequel.

Definition 2.4 Let $X$ be a real reflexive Banach space. The operator $A: X \rightarrow 2^{X^{*}}$ is called

(a) pseudomonotone if the following conditions hold:

(i) the set $A(u)$ is nonempty, bounded, closed and convex for all $u \in X$.

(ii) $A$ is upper semicontinuous from each finite-dimensional subspace of $X$ to the weak topology on $X^{*}$.

(iii) if $\left\{u_{n}\right\} \subset X$ with $u_{n} \rightarrow u$ in $X$ and $u_{n}^{*} \in A\left(u_{n}\right)$ are such that

$$
\limsup _{n \rightarrow \infty}\left\langle u_{n}^{*}, u_{n}-u\right\rangle_{X^{*} \times X} \leq 0,
$$

then to each element $v \in X$, there exists $u^{*}(v) \in A(u)$ with

$$
\left\langle u^{*}(v), u-v\right\rangle_{X^{*} \times X} \leq \liminf _{n \rightarrow \infty}\left\langle u_{n}^{*}, u_{n}-v\right\rangle_{X^{*} \times X} .
$$


(b) generalized pseudomonotone if the following holds: Let $\left\{u_{n}\right\} \subset X$ and $\left\{u_{n}^{*}\right\} \subset X^{*}$ with $u_{n}^{*} \in A\left(u_{n}\right)$. If $u_{n} \rightarrow u$ in $X$ and $u_{n}^{*} \rightarrow u^{*}$ in $X^{*}$ and

$$
\limsup _{n \rightarrow \infty}\left\langle u_{n}^{*}, u_{n}-u\right\rangle_{X^{*} \times X} \leq 0
$$

then the element $u^{*}$ lies in $A(u)$ and

$$
\left\langle u_{n}^{*}, u_{n}\right\rangle_{X^{*} \times X} \rightarrow\left\langle u^{*}, u\right\rangle_{X^{*} \times X}
$$

It is not difficult to see that every pseudomonotone operator is generalized pseudomonotone, see e.g., Carl-Le-Motreanu [30, Proposition 2.122] or GasińskiPapageorgiou [29, Proposition 1.4.11]. However, under the additional assumption of boundedness, we obtain the converse statement, see e.g., Carl-Le-Motreanu [30, Proposition 2.123] or Gasiński-Papageorgiou [29, Proposition 1.4.12].

Proposition 2.5 Let $X$ be a real reflexive Banach space and assume that $A: X \rightarrow 2^{X^{*}}$ satisfies the following conditions:

(i) for each $u \in X$ we have that $A(u)$ is a nonempty, closed and convex subset of $X^{*}$.

(ii) $A: X \rightarrow 2^{X^{*}}$ is bounded.

(iii) if $u_{n} \rightarrow u$ in $X$ and $u_{n}^{*} \rightarrow u^{*}$ in $X^{*}$ with $u_{n}^{*} \in A\left(u_{n}\right)$ and if

$$
\limsup _{n \rightarrow \infty}\left\langle u_{n}^{*}, u_{n}-u\right\rangle_{X^{*} \times X} \leq 0
$$

then $u^{*} \in A(u)$ and

$$
\left\langle u_{n}^{*}, u_{n}\right\rangle_{X^{*} \times X} \rightarrow\left\langle u^{*}, u\right\rangle_{X^{*} \times X}
$$

Then the operator $A: X \rightarrow 2^{X^{*}}$ is pseudomonotone.

Finally, we will state the following surjectivity theorem for multivalued mappings which are defined as the sum of a maximal monotone multivalued operator and a bounded multivalued pseudomonotone mapping. The following theorem can be found in Le [20, Theorem 2.2]. We use the notation $B_{R}(0):=\left\{u \in X:\|u\|_{X}<R\right\}$.

Theorem 2.6 Let $X$ be a real reflexive Banach space, let $F: D(F) \subset X \rightarrow 2^{X^{*}}$ be a maximal monotone operator, let $G: D(G)=X \rightarrow 2^{X^{*}}$ be a bounded multivalued pseudomonotone operator and let $L \in X^{*}$. Assume that there exist $u_{0} \in X$ and $R \geq\left\|u_{0}\right\|_{X}$ such that $D(F) \cap B_{R}(0) \neq \emptyset$ and

$$
\left\langle\xi+\eta-L, u-u_{0}\right\rangle_{X^{*} \times X}>0
$$

for all $u \in D(F)$ with $\|u\|_{X}=R$, all $\xi \in F(u)$ and all $\eta \in G(u)$. Then the inclusion

$$
F(u)+G(u) \ni L
$$

has a solution in $D(F)$. 


\section{Main Results}

Let us start this section with the assumption of the multivalued convection term $f: \Omega \times$ $\mathbb{R} \times \mathbb{R}^{N} \rightarrow 2^{\mathbb{R}}$ which will be needed in the existence result for problem (1.1). First of them provides general information on the regularity of $f$.

$H(f)_{0}: f: \Omega \times \mathbb{R} \times \mathbb{R}^{N} \rightarrow 2^{\mathbb{R}}$ has nonempty, compact and convex values; for all $(s, \xi) \in \mathbb{R} \times \mathbb{R}^{N}$, the multivalued mapping $x \mapsto f(x, s, \xi)$ has a measurable selection; for almost all $x \in \Omega$, the multivalued mapping $(s, \xi) \mapsto f(x, s, \xi)$ is upper semicontinuous.

Next two assumptions provide the growth conditions on $f$. In what follows by $p^{*}$ we denote the critical exponent corresponding to $p$, namely

$$
p^{*}:=\left\{\begin{array}{c}
\frac{N p}{N-p} \text { if } p<N \\
+\infty \text { if } p \geq N
\end{array}\right.
$$

$H(f)_{1}$ : there exists $\alpha \in L^{\frac{q_{1}}{q_{1}-1}}(\Omega), e_{1}, e_{2} \geq 0$ and $1<q_{1}<p^{*}$ such that

$$
|\eta| \leq e_{1}|\xi|^{p \frac{q_{1}-1}{q_{1}}}+e_{2}|s|^{q_{1}-1}+\alpha(x)
$$

for all $\eta \in f(x, s, \xi)$, for a.a. $x \in \Omega$, all $s \in \mathbb{R}$ and all $\xi \in \mathbb{R}^{N}$. $H(f)_{2}$ : there exist $w \in L_{+}^{1}(\Omega)$ and $b_{1}, b_{2} \geq 0$ are such that

$$
b_{1}+b_{2} \lambda_{1, p}^{-1}<\frac{a_{3}}{p-1}
$$

[see (2.3) for the definition of $a_{3}$ and (2.2) for the definition of $\lambda_{1, p}$ ] and

$$
\eta s \leq b_{1}|\xi|^{p}+b_{2}|s|^{p}+w(x)
$$

for all $\eta \in f(x, s, \xi)$, for a.a. $x \in \Omega$, all $s \in \mathbb{R}$ and all $\xi \in \mathbb{R}^{N}$.

We can provide an explicit example of a function $f$ satisfying the above hypotheses.

Example 3.1 For the simplicity we drop the $x$-dependence. Let $1<p<\infty, g: \mathbb{R}^{N} \rightarrow$ $\mathbb{R}$ be a continuous function and $h: \mathbb{R} \rightarrow \mathbb{R}$ a locally Lipschitz function such that there exists constants $e_{1}, e_{2}>0$ satisfying 
(i) $|g(\xi)| \leq e_{1}|\xi|^{p-1}$ for all $\xi \in \mathbb{R}^{N}$;

(ii) $\max _{\xi \in \partial h(s)}|\xi| \leq e_{2}|s|^{p-1}$ for all $s \in \mathbb{R}$,

where $\partial h$ stands for the generalized (Clarke) subdifferential of $h$. Let $a_{3}>\left(e_{2}+\right.$ $\left.\frac{1}{p}\right) \lambda_{1, p}^{-1}+\frac{e_{1}^{p^{\prime}}}{p^{\prime}}$. Then, it is straightforward to check that the function $f(s, \xi)=g(\xi)+$ $\partial h(s)$ satisfies hypotheses $H(f)_{0}-H(f)_{2}$ with respect to the weighted $p$-Laplacian and $q_{1}=p$, namely, $a(\xi)=\frac{a_{3}}{p-1}|\xi|^{p-2} \xi$ for all $\xi \in \mathbb{R}^{N}$.

Let $K$ be a subset of $W_{0}^{1, p}(\Omega)$ defined by

$$
K:=\left\{u \in W_{0}^{1, p}(\Omega) \mid u(x) \leq \Phi(x) \text { for a.a. } x \in \Omega\right\},
$$

where

$$
\Phi: \Omega \rightarrow[0,+\infty] \text { is a function. }
$$

It is obvious that the set $K$ is a nonempty, closed and convex subset of $W_{0}^{1, p}(\Omega)$.

Remark 3.2 From (3.2) it is clear that $0 \in K$.

The weak solutions for problem (1.1) are understood in the following sense.

Definition 3.3 We say that $u \in K$ is a weak solution of problem (1.1) if there exists $\eta \in L^{\frac{q_{1}}{q_{1}-1}}(\Omega)$ such that $\eta(x) \in f(x, u(x), \nabla u(x))$ for a.a. $x \in \Omega$ and

$$
\int_{\Omega}(a(x, \nabla u), \nabla(v-u))_{\mathbb{R}^{N}} d x=\int_{\Omega} \eta(x)(v(x)-u(x)) d x \text { for all } v \in K,
$$

where $K$ is given by (3.1).

The main result of this paper, providing existence of solutions as well as the properties of the solution set, is stated as the next theorem.

Theorem 3.4 Assume that hypotheses $H(a)_{0}-H(a)_{3}, H(f)_{0}-H(f)_{2}$ and (3.2) hold. Then the set of solutions of problem (1.1), $\mathcal{S}$, is nonempty, bounded and closed.

Proof The proof of the theorem is divided into three steps.

Step $1 . \mathcal{S} \neq \varnothing$ (i.e., problem (1.1) is solvable).

Consider the embedding operator $i: W_{0}^{1, p}(\Omega) \rightarrow L^{q_{1}}(\Omega)$ and denote by $i^{*}: L^{q_{1}^{\prime}}(\Omega) \rightarrow W_{0}^{1, p}(\Omega)^{*}$ its adjoint operator. As $1<q_{1}<p^{*}$, the embedding operator $i$ is compact and so is $i^{*}$. Moreover, by virtue of hypotheses $H(f)_{0}$ and $H(f)_{1}$, the Nemytskij operator $\tilde{N}_{f}: W_{0}^{1, p}(\Omega) \subset L^{q_{1}}(\Omega) \rightarrow 2^{L^{q_{1}^{\prime}}(\Omega)}$ associated to the multivalued mapping $f$ :

$$
\tilde{N}_{f}(u):=\left\{\eta \in L^{q_{1}^{\prime}}(\Omega) \mid \eta(x) \in f(x, u(x), \nabla u(x)) \text { for a.a. } x \in \Omega\right\}
$$

for all $u \in W_{0}^{1, p}(\Omega)$ is well-defined. 
Let $N_{f}:=i^{*} \circ \tilde{N}_{f}: W_{0}^{1, p}(\Omega) \rightarrow 2^{W_{0}^{1, p}(\Omega)^{*}}$ and introduce the indicator function $I_{K}: W_{0}^{1, p}(\Omega) \rightarrow \overline{\mathbb{R}}:=\mathbb{R} \cup\{+\infty\}$ of $K$, by

$$
I_{K}(u):= \begin{cases}0 & \text { if } u \in K \\ +\infty & \text { otherwise }\end{cases}
$$

It is easy to see that $u \in K$ is a weak solution of problem (1.1) (see Definition 3.3), if and only if $u$ solves the following inequality:

$$
\langle A(u)-\eta, v-u\rangle+I_{K}(v)-I_{K}(u) \geq 0 \quad \text { for all } v \in W_{0}^{1, p}(\Omega),
$$

with some $\eta \in N_{f}(u)$, where $A: W_{0}^{1, p}(\Omega) \rightarrow W_{0}^{1, p}(\Omega)^{*}$ is given by (2.4) and $\langle\cdot, \cdot\rangle$ stands for the duality pairing between $W_{0}^{1, p}(\Omega)^{*}$ and $W_{0}^{1, p}(\Omega)$. by

Next, let us consider the multivalued operator $\mathcal{A}: W_{0}^{1, p}(\Omega) \rightarrow 2^{W_{0}^{1, p}(\Omega)^{*}}$ defined

$$
\mathcal{A}(u)=A(u)-N_{f}(u) \text { for all } u \in W_{0}^{1, p}(\Omega) .
$$

Now we can reformulate problem (3.3) in the following equivalently way:

Find $u \in K$ such that

$$
\mathcal{A}(u)+\partial I_{K}(u) \ni 0
$$

where the notation $\partial I_{K}$ stands for the subdifferential of $I_{K}$ in the sense of convex analysis.

In order to prove that problem (3.4) has at least one weak solution, we will apply the surjectivity result for multivalued pseudomonotone operators (see Theorem 2.6). Let $u \in W_{0}^{1, p}(\Omega)$ and $\eta \in N_{f}(u)$ be arbitrary. By condition $H(f)_{1}$, we have

$$
\begin{aligned}
\|\eta\|_{W_{0}^{1, p}(\Omega)^{*}}^{q_{1}^{\prime}} & \leq\left\|i^{*}\right\|^{q_{1}^{\prime}}\|\xi\|_{L_{1}^{q_{1}^{\prime}(\Omega)}}^{q_{1}^{\prime}}=\left\|i^{*}\right\|^{q_{1}^{\prime}} \int_{\Omega}|\xi(x)|^{q_{1}^{\prime}} d x \\
& \leq C_{0} \int_{\Omega}\left(e_{1}|\nabla u(x)|^{p^{q_{1}-1}} \frac{e_{1}}{q_{1}}+\left.u(x)\right|^{q_{1}-1}+\alpha(x)\right)^{q_{1}^{\prime}} d x \\
& \leq C_{1}\left(\|\nabla u\|_{p}^{p}+\|u\|_{q_{1}}^{q_{1}}+\|\alpha\|_{q_{1}^{\prime}}^{q_{1}^{\prime}}\right),
\end{aligned}
$$

for some $C_{0}, C_{1}>0$, where $\xi \in \widetilde{N}_{f}(u)$ is such that $\eta=i^{*} \xi$. Remembering that $1<q_{1}<p^{*}$ and using Proposition 2.2 (or Lemma 2.1(ii)) we get that $\mathcal{A}: W_{0}^{1, p}(\Omega)$ $\rightarrow 2^{W_{0}^{1, p}(\Omega)^{*}}$ is a bounded mapping.

Next, using Proposition 2.5, we will prove that $\mathcal{A}$ is a pseudomonotone operator. By hypotheses on $f$, it is clear that $\mathcal{A}(u)$ is nonempty, closed and convex subset of $W^{1, p}(\Omega)^{*}$ for all $u \in W_{0}^{1, p}(\Omega)$. Moreover, as we just showed, $\mathcal{A}$ is a bounded 
mapping. So, it is enough to show that $\mathcal{A}$ is a generalized pseudomonotone operator (see Proposition 2.5).

Let $\left\{u_{n}\right\} \subset W_{0}^{1, p}(\Omega),\left\{u_{n}^{*}\right\} \subset W_{0}^{1, p}(\Omega)^{*}$ and $u \in W_{0}^{1, p}(\Omega)$ be such that

$$
\begin{aligned}
& u_{n} \rightarrow u \text { in } W_{0}^{1, p}(\Omega), u_{n}^{*} \rightarrow u^{*} \text { in } W_{0}^{1, p}(\Omega)^{*}, \\
& u_{n}^{*} \in \mathcal{A}\left(u_{n}\right) \text { for all } n \in \mathbb{N}, \\
& \limsup _{n \rightarrow \infty}\left\langle u_{n}^{*}, u_{n}-u\right\rangle \leq 0 .
\end{aligned}
$$

So, for each $n \in \mathbb{N}$, we are able to find an element $\xi_{n} \in \tilde{N}_{f}\left(u_{n}\right)$ such that $u_{n}^{*}=$ $A\left(u_{n}\right)-i^{*} \xi_{n}$. Because the embedding $W_{0}^{1, p}(\Omega) \rightarrow L^{q_{1}}(\Omega)$ is compact, from (3.6), we get that $u_{n} \rightarrow u$ in $L^{q_{1}}(\Omega)$. On the other hand, by virtue of (3.5), we have that the sequence $\left\{\xi_{n}\right\}$ is bounded in $L^{q_{1}^{\prime}}(\Omega)$. Therefore, by (3.8) we get

$$
\begin{aligned}
& \limsup _{n \rightarrow \infty}\left\langle A\left(u_{n}\right), u_{n}-u\right\rangle \\
& \quad=\limsup _{n \rightarrow \infty}\left\langle A\left(u_{n}\right), u_{n}-u\right\rangle-\underset{n \rightarrow \infty}{\limsup }\left\langle\xi_{n}, u_{n}-u\right\rangle_{L^{q_{1}}(\Omega)} \\
& \leq \limsup _{n \rightarrow \infty}\left\langle A\left(u_{n}\right)-i^{*} \xi_{n}, u_{n}-u\right\rangle \\
& =\limsup _{n \rightarrow \infty}\left\langle u_{n}^{*}, u_{n}-u\right\rangle \leq 0
\end{aligned}
$$

This fact together with (3.6) and the $\left(\mathrm{S}_{+}\right)$-property of $A$ (see Proposition 2.2), imply that $u_{n} \rightarrow u$ in $W_{0}^{1, p}(\Omega)$ and by the continuity of $A$ (see Lemma 2.1(i)), we have

$$
\left\langle u_{n}^{*}, u_{n}\right\rangle \rightarrow\left\langle u^{*}, u\right\rangle \text { and } A\left(u_{n}\right) \rightarrow A(u) \text { in } W_{0}^{1, p}(\Omega)^{*} .
$$

As $\xi_{n} \in \widetilde{N}_{f}\left(u_{n}\right)$, we have

$$
\xi_{n}(x) \in f\left(x, u_{n}(x), \nabla u_{n}(x)\right) \text { for a.a. } x \in \Omega \text {. }
$$

Estimate (3.5) and convergence (3.6) imply that the sequence $\left\{\xi_{n}\right\}$ is bounded in $L^{q_{1}^{\prime}}(\Omega)$. Passing to a subsequence if necessary, we may assume that

$$
\xi_{n} \rightarrow \xi \text { in } L^{q_{1}^{\prime}}(\Omega)
$$

for some $\xi \in L^{q_{1}^{\prime}}(\Omega)$. Recall that $u_{n} \rightarrow u$ in $W_{0}^{1, p}(\Omega)$, so, passing to a subsequence if necessary, we have

$$
u_{n}(x) \rightarrow u(x) \text { and } \nabla u_{n}(x) \rightarrow \nabla u(x) \text { as } n \rightarrow \infty \text { for a.e. } x \in \Omega \text {. }
$$

Since $\mathbb{R} \times \mathbb{R}^{n} \ni(s, w) \mapsto f(x, s, w) \subset \mathbb{R}^{N}$ is upper semicontinuous and has nonempty closed convex values (see hypotheses $H(f)_{0}$ ), it follows from Theorem 7.2.2 of Aubin and Frankowska [31, p. 273] that

$$
\xi(x) \in f(x, u(x), \nabla u(x)) \text { for a.a. } x \in \Omega \text {. }
$$


Thus $\xi \in \tilde{N}_{f}(u)$ and $i^{*} \xi \in N_{f}(u)$. Therefore, we obtain that

$$
u^{*}=A(u)-i^{*} \xi \in \mathcal{A}(u)
$$

which implies that $\mathcal{A}$ is generalized pseudomonotone.

Because $\mathcal{A}$ is a bounded operator with nonempty, closed and convex values, by Proposition 2.5 we conclude that $\mathcal{A}$ is a pseudomonotone operator.

Next, we will show the existence of a constant $R>0$ such that

$$
\left\langle u^{*}+\eta, u\right\rangle>0
$$

for all $u^{*} \in \mathcal{A}(u)$, all $\eta \in \partial I_{K}(u)$ and all $u \in W_{0}^{1, p}(\Omega)$ with $\|u\|=R$.

For any $u^{*} \in \mathcal{A}(u)$, we can find $\xi \in \widetilde{N}_{f}(u)$ such that $u^{*}=A(u)-i^{*} \xi$. As $0 \in K$ (see Lemma 2.1(iii)), we have

$$
\begin{aligned}
\left\langle u^{*}+\eta, u\right\rangle & \geq \int_{\Omega}(a(x, \nabla u), \nabla u)_{\mathbb{R}^{N}} d x-\int_{\Omega} \xi(x) u(x) d x+I_{K}(u)-I_{K}(0) \\
& \geq \frac{a_{3}}{p-1}\|\nabla u\|_{p}^{p}-\int_{\Omega} \xi(x) u(x) d x+I_{K}(u) .
\end{aligned}
$$

Because $I_{K}: W_{0}^{1, p}(\Omega) \rightarrow \overline{\mathbb{R}}$ is a proper, convex and lower semicontinuous function, we can apply Proposition 1.10 in Brézis [32], and obtain that

$$
I_{K}(v) \geq-\alpha_{K}\|v\|-\beta_{K} \text { for all } v \in W_{0}^{1, p}(\Omega)
$$

for some $\alpha_{K}, \beta_{K}>0$. Moreover, by hypothesis $H(f)_{2}$, we have that

$$
\int_{\Omega} \xi(x) u(x) d x \leq b_{1}\|\nabla u\|_{p}^{p}+b_{2}\|u\|_{p}^{p}+\|w\|_{1} .
$$

Using (3.14), (3.15) and the inequality

$$
\|u\|_{p}^{p} \leq \lambda_{1, p}^{-1}\|\nabla u\|_{p}^{p} \quad \text { for all } u \in W_{0}^{1, p}(\Omega)
$$

[(see (2.2)], in (3.13), we obtain

$$
\begin{aligned}
& \left\langle u^{*}+\eta, u\right\rangle \\
& \quad \geq \frac{a_{3}}{p-1}\|\nabla u\|_{p}^{p}-b_{1}\|\nabla u\|_{p}^{p}-b_{2}\|u\|_{p}^{p}-\|w\|_{1}-\alpha_{K}\|u\|-\beta_{K} \\
& \quad \geq\left(\frac{a_{3}}{p-1}-b_{1}-b_{2} \lambda_{1, p}^{-1}\right)\|u\|^{p}-\|w\|_{1}-\alpha_{K}\|u\|-\beta_{K} .
\end{aligned}
$$


As $b_{1}+b_{2} \lambda_{1, p}^{-1}<\frac{a_{3}}{p-1}$ (see hypotheses $H(f)_{2}$ ), we can find $R_{0}>0$ large enough such that for all $R \geq R_{0}$ we have

$$
\left(\frac{a_{3}}{p-1}-b_{1}-b_{2} \lambda_{1, p}^{-1}\right) R^{p}-\|w\|_{1}-\alpha_{K} R-\beta_{K}>0 .
$$

Therefore, inequality (3.12) holds.

Because $\partial I_{K}: W_{0}^{1, p}(\Omega) \rightarrow 2^{W_{0}^{1, p}(\Omega)^{*}}$ is a maximal monotone operator, we can apply Theorem 2.6 with $F=\partial I_{K}, G=\mathcal{A}, L=0$, and conclude that inclusion (3.4) has at least one solution $u \in K$ which is a solution of (3.3) and so also a solution of (1.1) in the sense of Definition 3.3. Thus, $\mathcal{S} \neq \varnothing$.

Step 2. The set $\mathcal{S}$ is closed in $W_{0}^{1, p}(\Omega)$.

Let $\left\{u_{n}\right\} \subset \mathcal{S}$ be a sequence such that

$$
u_{n} \rightarrow u \text { in } W_{0}^{1, p}(\Omega)
$$

for some $u \in W_{0}^{1, p}(\Omega)$. For each $n \in \mathbb{N}$, we can find $\xi_{n} \in \widetilde{N}_{f}\left(u_{n}\right)$ such that

$$
\left\langle A\left(u_{n}\right), v-u_{n}\right\rangle+\left\langle\xi_{n}, v-u_{n}\right\rangle_{L^{q_{1}(\Omega)}}+I_{K}(v)-I_{K}\left(u_{n}\right) \geq 0
$$

for all $v \in W_{0}^{1, p}(\Omega)$. By hypothesis $H(f)_{1}$ and (3.16) we know that $\left\{\xi_{n}\right\}$ is bounded in $L^{q_{1}^{\prime}}(\Omega)$. So, passing to a subsequence if necessary, we may assume that

$$
\xi_{n} \rightarrow \xi \text { in } L^{q_{1}^{\prime}}(\Omega) .
$$

As before, using Theorem 7.2.2 of Aubin and Frankowska [31, p. 273], we obtain that

$$
\xi(x) \in f(x, u(x), \nabla u(x)) \text { for a.a. } x \in \Omega,
$$

i.e., $\xi \in \widetilde{N}_{f}(u)$. Passing to the upper limit in (3.17) as $n \rightarrow \infty$ and using the lower semicontinuity of $I_{K}$ we conclude that $u \in K$ is a solution of problem (1.1). Thus, the set $\mathcal{S}$ is closed.

Step 3. The set $\mathcal{S}$ is bounded.

If the set $K$ is bounded the result is clearly true. So, let us assume that $K$ be unbounded. Proceeding by contradiction, assume that the set $\mathcal{S}$ is unbounded, so we can find a sequence $\left\{u_{n}\right\} \subseteq \mathcal{S}$ such that

$$
\left\|u_{n}\right\| \rightarrow+\infty
$$

Similarly as in (3.13), we show that

$$
0 \geq\left\langle A\left(u_{n}\right)-i^{*} \xi_{n}, u_{n}\right\rangle
$$




$$
\geq\left(\frac{a_{3}}{p-1}-b_{1}-b_{2} \lambda_{1, p}^{-1}\right)\left\|u_{n}\right\|^{p}-\|w\|_{1}-\alpha_{K}\left\|u_{n}\right\|-\beta_{K},
$$

for some $\xi_{n} \in \tilde{N}_{f}\left(u_{n}\right)$. This inequality together with (3.18) give a contradiction. Therefore, the set $\mathcal{S}$ is bounded.

Acknowledgements We would like to thank the anonymous referee's remarks which increase the content of the paper considerably. This project has received funding from the NNSF of China Grant Nos. 12001478, 12026255 and 12026256, and the European Union's Horizon 2020 Research and Innovation Programme under the Marie Sklodowska-Curie grant agreement No. 823731 CONMECH, National Science Center of Poland under Preludium Project No. 2017/25/N/ST1/00611, the Startup Project of Doctor Scientific Research of Yulin Normal University No. G2020ZK07, the Natural Science Foundation of Guangxi Grant No. 2021GXNSFFA196004 and 2020GXNSFBA297137 and the International Project co-financed by the Ministry of Science and Higher Education of Republic of Poland under Grant No. 3792/GGPJ/H2020/2017/0.

Open Access This article is licensed under a Creative Commons Attribution 4.0 International License, which permits use, sharing, adaptation, distribution and reproduction in any medium or format, as long as you give appropriate credit to the original author(s) and the source, provide a link to the Creative Commons licence, and indicate if changes were made. The images or other third party material in this article are included in the article's Creative Commons licence, unless indicated otherwise in a credit line to the material. If material is not included in the article's Creative Commons licence and your intended use is not permitted by statutory regulation or exceeds the permitted use, you will need to obtain permission directly from the copyright holder. To view a copy of this licence, visit http://creativecommons.org/licenses/by/4.0/.

\section{References}

1. Averna, D., Motreanu, D., Tornatore, E.: Existence and asymptotic properties for quasilinear elliptic equations with gradient dependence. Appl. Math. Lett. 61, 102-107 (2016)

2. Bai, Y.R.: Existence of solutions to nonhomogeneous Dirichlet problems with dependence on the gradient. Electron. J. Differ. Equ. 101(18), (2018) pp. 1-18

3. Bai, Y.R., Gasiński, L., Papageorgiou, N.S.: Nonlinear nonhomogeneous Robin problems with dependence on the gradient. Bound Value Probl. 17, (2018). https://doi.org/10.1186/s13661-018-0936-8

4. Faraci, F., Motreanu, D., Puglisi, D.: Positive solutions of quasi-linear elliptic equations with dependence on the gradient. Cal. Var. PDEs 54(1), 525-538 (2015)

5. Gasiński, L., Papageorgiou, N.S.: On nonlinear elliptic hemivariational inequalities of second order. Acta Math. Sci. Ser. B (Engl. Ed.) 24(3), 451-462 (2004)

6. Gasiński, L., Papageorgiou, N.S.: Positive solutions for nonlinear elliptic problems with dependence on the gradient. J. Differ. Equ. 263, 1451-1476 (2017)

7. Gasiński, L., Winkert, P.: Existence and uniqueness results for double phase problems with convection term. J. Differ. Equ. 268(8), 4183-4193 (2020)

8. Motreanu, D., Motreanu, V.V., Moussaoui, A.: Location of nodal solutions for quasilinear elliptic equations with gradient dependence. Discrete Contin. Dyn. Syst. Ser. S 11(2), 293-307 (2018)

9. Guarnotta, U., Marano, S.A., Motreanu, D.: On a singular Robin problem with convection terms. Adv. Nonlinear Stud. 20(4), 895-909 (2020)

10. Papageorgiou, N.S., Rădulescu, V.D., Repovš, D.D.: Nonlinear elliptic inclusions with unilateral constraint and dependence on the gradient. Appl. Math. Optim. 78(1), 1-23 (2018)

11. Faraci, F., Puglisi, D.: A singular semilinear problem with dependence on the gradient. J. Differ. Equ. 260(4), 3327-3349 (2016)

12. Figueiredo, G.M., Madeira, G.F.: Positive maximal and minimal solutions for non-homogeneous elliptic equations depending on the gradient. J. Differ. Equ. 274, 857-875 (2021)

13. Papageorgiou, N.S., Rădulescu, V.D., Repov̌̌, D.D.: Positive solutions for nonlinear Neumann problems with singular terms and convection. J. Math. Pures Appl. 136, 1-21 (2020) 
14. Tanaka, M.: Existence of a positive solution for quasilinear elliptic equations with nonlinearity including the gradient. Bound Value Probl. 2013, 173 (2013)

15. Guarnotta, U., Marano, S.A.: Infinitely many solutions to singular convective Neumann systems with arbitrarily growing reactions. J. Differ. Equ. 271, 849-863 (2021)

16. Liu, Z.H., Motreanu, D., Zeng, S.D.: Positive solutions for nonlinear singular elliptic equations of p-Laplacian type with dependence on the gradient. Cal. Var. PDEs 58(1), 22 (2019)

17. Marano, S.A., Winkert, P.: On a quasilinear elliptic problem with convection term and nonlinear boundary condition. Nonlinear Anal. 187, 159-169 (2019)

18. de Araujo, A.L.A., Faria, L.F.O.: Positive solutions of quasilinear elliptic equations with exponential nonlinearity combined with convection term. J. Differ. Equ. 267(8), 4589-4608 (2019)

19. Bai, Y.R., Papageorgiou, N.S., Zeng, S.D.: A singular eigenvalue problem for the Dirichlet $(p, q)$ Laplacian. Math. Z. (2021). https://doi.org/10.1007/s00209-021-02803-w

20. Le, V.K.: A range and existence theorem for pseudomonotone perturbations of maximal monotone operators. Proc. Am. Math. Soc. 139(5), 1645-1658 (2011)

21. Carl, S., Le, V.K.: Multi-valued variational inequalities and inclusions. Springer, Cham (2021)

22. Motreanu, D., Winkert, P.: Existence and asymptotic properties for quasilinear elliptic equations with gradient dependence. Appl. Math. Lett. 95, 78-84 (2019)

23. Zeng, S.D., Gasiński, L., Winkert, P., Bai, Y.R.: Existence of solutions for double phase obstacle problems with multivalued convection term. J. Math. Anal. Appl. 501, 123997 (2021)

24. Zeng, S.D., Bai, Y.R., Gasiński, L., Winkert, P.: Existence results for double phase implicit obstacle problems involving multivalued operators. Cal. Var. PDEs 59(5), 18 (2020)

25. Zeng, S.D., Bai, Y.R., Gasiński, L., Winkert, P.: Convergence analysis for double phase obstacle problems with multivalued convection term. Adv. Nonlinear Anal. 10, 659-672 (2021)

26. Mingione, G., Rădulescu, V.D.: Recent developments in problems with nonstandard growth and nonuniform ellipticity. J. Math. Anal. Appl. 501, 125197 (2021)

27. Lê, A.: Eigenvalue problems for the $p$-Laplacian. Nonlinear Anal. 64(5), 1057-1099 (2006)

28. Gasiński, L., Papageorgiou, N.S.: Exercises in analysis. Part 1: nonlinear analysis. Springer, Heidelberg (2014)

29. Gasiński, L., Papageorgiou, N.S.: Nonsmooth critical point theory and nonlinear boundary value problems. Chapman \& Hall/CRC, Boca Raton (2005)

30. Carl, S., Le, V.K., Motreanu, D.: Nonsmooth variational problems and their inequalities. Springer, New York (2007)

31. Aubin, J.P., Frankowska, H.: Set-valued analysis. Birkhäuser, Boston (1990)

32. Brézis, H.: Functional analysis, Sobolev spaces and partial differential equations. Springer, New York (2011)

Publisher's Note Springer Nature remains neutral with regard to jurisdictional claims in published maps and institutional affiliations. 